\title{
PARTIAL REGULARITY OF WEAK SOLUTIONS TO MAXWELL'S EQUATIONS IN A QUASI-STATIC ELECTROMAGNETIC FIELD*
}

\author{
MIN-CHUN HONG ${ }^{\dagger}$, YOSHIHIRO TONEGAWA AND ALZUBAIDI YASSIN ${ }^{\dagger}$ \\ Delicated to Professor Neil Trudinger on the occasion of his 65th birthday
}

\begin{abstract}
We study Maxwell's equations in a quasi-static electromagnetic field, where the electrical conductivity of the material depends on the temperature. By establishing the reverse Hölder inequality, we prove partial regularity of weak solutions to the non-linear elliptic system and the non-linear parabolic system in a quasi-static electromagnetic field.
\end{abstract}

Key words. Partial regularity, elliptic systems, parabolic systems.

AMS subject classifications. 35J45, 35J60, 58E20

1. Introduction. In this paper, let $\Omega$ be a domain in $\mathbb{R}^{n}$ with $n \geq 3$, and let $u(x)$ and $H^{i}(x)$ for $i=1, \ldots, n$ be scalar functions defined on $\Omega$. For any positive integer $k$, let $\Lambda_{k}(\Omega)$ denote the space of $k$-forms on $\Omega$. We have the usual exterior derivative $d$ of forms with $d: \Lambda_{k}(\Omega) \rightarrow \Lambda_{k+1}(\Omega)$. Consider a 1-form $H=\sum_{i=1}^{n} H^{i}(x) d x_{i}$, which may be regarded as a connection in differential geometry. We define the curvature $F$ of the connection $H$ by

$$
F=d H=\sum_{i<j} F^{i j} d x_{i} \wedge d x_{j}
$$

where $F^{i j}=\frac{\partial H^{j}}{\partial x_{i}}-\frac{\partial H^{i}}{\partial x_{j}}$ (e.g. [9]).

Let $*$ be the Hodge star linear operator which assigns to each $k$-form on $\Omega$ an $(n-k)$-form and which satisfies

$$
* *=(-1)^{k(n-k)} .
$$

We have a product $\langle\cdot, \cdot\rangle$ in the $k$-form space $\Lambda_{k}(\Omega)$

$$
\langle a, b\rangle d x_{1} \wedge \ldots \wedge d x_{n}=a \wedge * b, \quad|a|^{2}=\langle a, a\rangle
$$

for all $a, b \in \Lambda_{k}(\Omega)$ (e.g. [15]).

By definition, we have

$$
|H|^{2}=\langle H, H\rangle=\sum_{i=1}^{n}\left(H^{i}\right)^{2}, \quad|d H|^{2}=\langle d H, d H\rangle=\frac{1}{2} \sum_{i, j=1}^{n}\left(F^{i j}\right)^{2} .
$$
and

Let $d^{*}$ be the adjoint operator of $d$ with $d^{*}=(-1)^{n+n k+1} * d *: \Lambda_{k}(\Omega) \rightarrow \Lambda_{k-1}(\Omega)$

$$
\int_{\Omega}\langle d a, b\rangle d x=\int_{\Omega}\left\langle a, d^{*} b\right\rangle d x
$$

for $a \in \Lambda_{k}(\Omega), b \in \Lambda_{k+1}(\Omega)$, where $b$ or $a$ has compact support inside of $\Omega$.

\footnotetext{
* Received April 6, 2008; accepted for publication June 13, 2008.

${ }^{\dagger}$ Department of Mathematics, University of Queensland, Brisbane, QLD 4702, Australia (hong @maths.uq.edu.au; yassin@maths.uq.edu.au).

${ }^{\ddagger}$ Department of Mathematics, Hokkaido University, Sapporo 060-0810, Japan (tonegawa@math. sci.hokudai.ac.jp).
} 
We consider the following system

$$
\begin{gathered}
d^{*}[\sigma(u) d H]=0 \quad \text { in } \Omega \\
-\triangle u=\sigma(u)|d H|^{2} \quad \text { in } \Omega
\end{gathered}
$$

where $\sigma$ is a positive function defined on $\mathbb{R}$.

We say that a pair $(u, H)$ is a weak solution to the system (1.1)-(1.2) if $u \in$ $W^{1, q}(\Omega)$ for some $q \in\left(1, \frac{n}{n-1}\right)$ and $H \in W^{1,2}\left(\Omega ; \mathbb{R}^{n}\right)$, and the pair $(u, H)$ satisfies the following:

$$
\begin{gathered}
\int_{\Omega}\langle\sigma(u) d H, d \phi\rangle d x=0, \\
\int_{\Omega} \nabla u \cdot \nabla \psi d x=\int_{\Omega} \sigma(u)|d H|^{2} \psi d x
\end{gathered}
$$

for all $\phi:=\sum_{i=1}^{n} \phi^{i}(x) d x_{i}$ for $i=1, \ldots, n$, where $\phi^{i} \in C_{0}^{2}(\Omega ; \mathbb{R})$ and $\psi \in C_{0}^{2}(\Omega ; \mathbb{R})$.

Assumption $(\mathrm{S}) . \sigma(u)$ is uniformly Hölder continuous in $\mathbb{R}$ and there exist two constants $\sigma_{1}$ and $\sigma_{1}$ such that

$$
0<\sigma_{1} \leq \sigma(u) \leq \sigma_{2} .
$$

Uniform Hölder continuity above can be replaced by the assumption of Hölder continuity of $\sigma(u)$ (see [1]). Without loss of generality, we assume that Assumption (S) holds throughout this paper.

In this paper, we prove the partial regularity of the above weak solution to the system (1.1)-(1.2) in the following:

THEOREM A. Let a pair $(u, H)$ be a weak solution to the system (1.1)-(1.2) with $u \in W^{1, q}(\Omega, \mathbb{R})$ for some $q \in\left(1, \frac{n}{n-1}\right), H \in W^{1,2}\left(\Omega ; \mathbb{R}^{n}\right)$ and $d^{*} H(x)=0$ for a.e. $x \in \Omega$. Then there exists an open subset $\Omega_{0}$ of $\Omega$ such that the solution $(u, H)$ is $C^{1, \alpha}$ locally in $\Omega_{0}$, and $\mathcal{H}^{n-q_{1}}\left(\Omega \backslash \Omega_{0}\right)=0$ for some $q_{1}>\frac{n}{n-1}$, where $\mathcal{H}^{n-q_{1}}$ denotes the $\left(n-q_{1}\right)$-dimensional Hausdorff measure.

The system (1.1)-(1.2) is not elliptic since it is invariant under the gauge transformation $(u, H) \rightarrow(u, H+\nabla \xi)$ for all $\xi \in W^{2,2}(\Omega)$. By a gauge transformation, one can fix a gauge satisfying

$$
d^{*} H=\operatorname{div} H=\sum_{i} \frac{\partial H^{i}}{\partial x_{i}}=0
$$

The system (1.1)-(1.2) with $d^{*} H=0$ on $\Omega$ is a quasi-linear elliptic system which has a natural growth structure. When $n=3$, Yin in [13], [14] proved the existence of weak solutions $(u, H)$ to $(1.1)-(1.2)$ with $u \in W^{1, q}(\Omega, \mathbb{R}), q \in\left(1, \frac{n}{n-1}\right), H \in W^{1,2}\left(\Omega ; \mathbb{R}^{3}\right)$ and $\operatorname{div} H=0$ in $\Omega$. Moreover, he also proved the regularity of continuous weak solutions to (1.1)-(1.2). However, he also pointed out that the continuity of the weak solution is unknown. For $n>3$, we have a similar existence result for weak solutions to the system (1.1)-(1.2) using the same proof as in [13] and [14]. Generally, weak solutions of non-linear elliptic systems may have singularities by De Giorgi's example and Giusti-Miranda's example (see [8]). Partial regularity theory for weak solutions of non-linear elliptic systems began around 1968 by Morrey, Giusti-Miranda (e.g. see [1] or [2]). The reader may refer to an excellent book [1] on the further development of the general theory of partial regularity. For many cases of quasi-linear elliptic systems 
which have natural growth, e.g. harmonic map equations, one usually assumes that weak solutions to (1.1)-(1.2) are in the space $W^{1,2} \cap L^{\infty}(\Omega)$. From the existence result for weak solutions, we only know $u \in W^{1, q}(\Omega)$ with $q \in\left(1, \frac{n}{n-1}\right)$, we do not know if $u$ in $W^{1,2} \cap L^{\infty}(\Omega)$, so the general theory of non-linear elliptic systems in [1] does not apply to our system (1.1)-(1.2). Recently, the partial regularity of non-linear elliptic systems involving forms and maps was studied in [4].

When $n=3$, the system (1.1)-(1.2) arises from approximating Maxwell's equations in a quasi-stationary electromagnetic field with non-ferromagnetic bodies (e.g. [11]). In the study of the penetration of a magnetic field in a medium, the electrical resistance strongly depends on the temperature. By taking the temperature effect into consideration, the classical Maxwell system in the quasi-static electromagnetic field can be reduced to the following system (see [11], [13] and [14]):

$$
\begin{gathered}
\partial_{t} H+\nabla \times[\sigma(u) \nabla \times H]=0 ; \quad(x, t) \in \Omega \times(0, T) \\
\partial_{t} u-\triangle u=\sigma(u)|\nabla \times H|^{2} ; \quad(x, t) \in \Omega \times(0, T) \\
\operatorname{div} H=0 ; \quad(x, t) \in \Omega \times(0, T),
\end{gathered}
$$

where $H=\left(H^{1}(x, t), H^{2}(x, t), H^{3}(x, t)\right)$ and $u(x, t)$ represent the strength of the magnetic field and the temperature respectively, and $\sigma^{-1}(u)$ denotes the electrical conductivity of the material. By changing the notation from vector functions to forms, we can consider the vector function $H$ and its 'curl' $\nabla \times \tilde{H}$ as a 1-form $H(x)$ and its curvature $d H$ respectively.

Now we generalize the Maxwell systems (1.3)-(1.5) to higher dimensional cases; i.e $n>3$. Let $u=u(x, t)$ and $H=\sum_{i} H^{i}(x, t) d x_{i}$ be a function and a 1 -form on $Q_{T}=\Omega \times[0, T]$ respectively. Then we consider the following system

$$
\begin{gathered}
\partial_{t} H=-d^{*}[\sigma(u) d H] ; \quad \text { in } Q_{T} \\
\partial_{t} u=\triangle u+\sigma(u)|d H|^{2} ; \text { in } Q_{T},
\end{gathered}
$$

with $d^{*} H(x, t)=0$ for a. e. $(x, t) \in Q_{T}$, where $\sigma$ is a positive function satisfying Assumption (S). The weak solution in $V_{q}^{1,0}\left(Q_{T}\right)$ to system (1.6)-(1.7) is defined in Section 4.

The second main result of this paper is the following:

THEOREM B. Let $(u, H)$ be a weak solution to equations (1.6) and (1.7) with $u \in V_{q}^{1,0}\left(Q_{T}\right)$ for some $q \in\left(1, \frac{n+2}{n+1}\right), H^{i} \in V_{2}^{1,0}\left(Q_{T} ; \mathbb{R}^{n}\right)$ for $i=1, \ldots, n$ and $d^{*} H=0$ for a. e. $(x, t) \in Q_{T}$. Then when $n \geq 3$, there exists an open subset $\tilde{Q}$ of $Q_{T}$ such that the solution $(u, H)$ is $C^{1, \alpha}$ in $\tilde{Q}$, and $\mathcal{H}^{n+2-q_{3}}\left(Q_{T} \backslash \tilde{Q}\right)=0$ with $q_{3}=\frac{(n+2) p}{n+2-2 p}$ for some $p>2$, where $\mathcal{H}^{n+2-q_{3}}$ denotes the Hausdorff measure.

The paper is organized as follows. In Section 2, we prove Caccioppoli's inequality for $H$ (Lemma 1 ) and then obtain $L^{p}$-estimates (Theorem 3 ) by applying the reverse Hölder inequality. In Section 3, we prove partial regularity for system (1.1)-(1.2) by applying Theorem 3. Finally, in Section 4, we establish partial regularity of weak solutions for the parabolic problem (1.6)-(1.7)using the analogous techniques as in the elliptic case. 
2. Reverse Hölder inequalities and $L^{p}$-estimates. In this section, we establish the Caccioppoli inequality for $H$ and the $L^{p}$-estimate.

Let $x_{0}$ be a point in $\Omega$ with $B_{R}\left(x_{0}\right) \subset \Omega$. For any function $f$, any 1-form $H$ and any measurable set $A$, denote

$$
f_{A} f d x=\frac{1}{|A|} \int_{A} f d x, \quad f_{x_{0}, R}=f_{B_{R}\left(x_{0}\right)} f d x, \quad(H)_{x_{0}, R}=H_{x_{0}, R}^{i} d x_{i} .
$$

Lemma 1. (Caccioppoli's inequality for $H$ ) Assume that $(u, H)$ is a weak solution of (1.1)-(1.2) with $u \in W^{1, q}, H \in W^{1,2}$ and $d^{*} H(x)=0$ for a.e. $x \in \Omega$. Then there exists a constant $C$ such that for any $x_{0} \in \Omega$ and $\rho, R$ with $\rho<R$ with $B_{R}\left(x_{0}\right) \subset \Omega$,

$$
\int_{B_{\rho}\left(x_{0}\right)}|\nabla H|^{2} d x \leq \frac{C}{(R-\rho)^{2}} \int_{B_{R}\left(x_{0}\right)}\left|H-(H)_{x_{0}, R}\right|^{2} d x
$$

Proof. Without loss of generality, we assume $x_{0}=0$. Let $\phi$ be a smooth cut-off function with $\phi=1$ on $B_{\rho}, \phi=0$ outside $B_{R},|\phi| \leq 1$ on $B_{R} \backslash B_{\rho}$, and $|\nabla \phi| \leq \frac{C}{R-\rho}$ on $B_{R} \backslash B_{\rho}$. Choosing $\phi^{2}\left(H-H_{0, R}\right)$ as a test function in (1.1), we have

$$
\int_{B_{R}}\left\langle d^{*}[\sigma(u) d H], \phi^{2}\left(H-H_{0, R}\right)\right\rangle d x=0
$$

By Stokes' formula, we obtain

$$
\begin{aligned}
\int_{B_{R}} \sigma(u)|d H|^{2} \phi^{2} d x & =-2 \int_{B_{R}}\left\langle\sigma(u) d H, \phi d \phi \wedge\left(H-H_{0, R}\right)\right\rangle d x \\
& \leq \varepsilon \int_{B_{R}}|d H|^{2} \phi^{2} d x+\frac{C}{(R-\rho)^{2}} \int_{B_{R}}\left|H-H_{0, R}\right|^{2} d x
\end{aligned}
$$

Choosing $\varepsilon$ to be sufficiently small, we have

$$
\int_{B_{R}}|d H|^{2} \phi^{2} d x \leq \frac{C}{(R-\rho)^{2}} \int_{B_{R}}\left|H-H_{0, R}\right|^{2} d x
$$

We note

$$
\begin{aligned}
|d H|^{2} & =\frac{1}{2} \sum_{i, j=1}^{n}\left(\frac{\partial H^{i}}{\partial x_{j}}-\frac{\partial H^{j}}{\partial x_{i}}\right)^{2} \\
& =|\nabla H|^{2}-\sum_{i, j=1}^{n} \frac{\partial H^{i}}{\partial x_{j}} \frac{\partial H^{j}}{\partial x_{i}}
\end{aligned}
$$

Since $H \in W^{1,2}$, we can approximate it by smooth functions $H_{k}$ in $W^{1,2}$ for 
$k=1,2,3, \cdots$. By Stokes' formula, we have

$$
\begin{aligned}
\int_{B_{R}}\left|d H_{k}\right|^{2} \phi^{2} d x & =\int_{B_{R}}\left|\nabla H_{k}\right|^{2} \phi^{2} d x-\sum_{i, j=1}^{n} \int_{B_{R}} \frac{\partial H_{k}^{i}}{\partial x_{j}} \frac{\partial H_{k}^{j}}{\partial x_{i}} \phi^{2} d x \\
& =\int_{B_{R}}\left|\nabla H_{k}\right|^{2} \phi^{2} d x+2 \sum_{i, j=1}^{n} \int_{B_{R}} \frac{\partial H_{k}^{i}}{\partial x_{j}} \phi \frac{\partial \phi}{\partial x_{i}}\left[H_{k}^{j}-\left(H_{k}^{j}\right)_{0, R}\right] d x \\
& +\int_{B_{R}} \sum_{i, j=1}^{n} \phi^{2}\left[H_{k}^{j}-\left(H_{k}^{j}\right)_{0, R}\right] \frac{\partial^{2} H_{k}^{i}}{\partial x_{j} \partial x_{i}} d x \\
& =\int_{B_{R}}\left|\nabla H_{k}\right|^{2} \phi^{2} d x+2 \sum_{i, j=1}^{n} \int_{B_{R}} \frac{\partial H_{k}^{i}}{\partial x_{j}} \phi \frac{\partial \phi}{\partial x_{i}}\left[H_{k}^{j}-\left(H_{k}^{j}\right)_{0, R}\right] d x \\
& -\int_{B_{R}} \sum_{i, j=1}^{n} \frac{\partial}{\partial x_{j}}\left(\phi^{2}\left[H_{k}^{j}-\left(H_{k}^{j}\right)_{0, R}\right]\right) \frac{\partial H_{k}^{i}}{\partial x_{i}} d x
\end{aligned}
$$

where we note $\frac{\partial^{2} H_{k}^{i}}{\partial x_{j} \partial x_{i}}=\frac{\partial^{2} H_{k}^{i}}{\partial x_{i} \partial x_{j}}$. As $k \rightarrow \infty$, it follows from using $\sum_{i} \frac{\partial H^{i}}{\partial x_{i}}=0$ that

$$
\int_{B_{R}}|d H|^{2} \phi^{2} d x=\int_{B_{R}}|\nabla H|^{2} \phi^{2} d x+2 \sum_{i, j=1}^{n} \int_{B_{R}} \frac{\partial H^{i}}{\partial x_{j}} \phi \frac{\partial \phi}{\partial x_{i}}\left[H^{j}-\left(H^{j}\right)_{0, R}\right] d x
$$

Therefore

$$
\begin{aligned}
\int_{B_{R}}|\nabla H|^{2} \phi^{2} d x \leq & \int_{B_{R}}|d H|^{2} \phi^{2} d x+\frac{1}{2} \int_{B_{R}}|\nabla H|^{2} \phi^{2} d x \\
& +\frac{C}{(R-\rho)^{2}} \int_{B_{R}}\left|H-(H)_{0, R}\right|^{2} d x .
\end{aligned}
$$

Now it follows from (2.1) that

$$
\int_{B_{R}}|\nabla H|^{2} \phi^{2} d x \leq \frac{C}{(R-\rho)^{2}} \int_{B_{R}}\left|H-(H)_{0, R}\right|^{2} d x .
$$

This proves our claim.

By the Proposition in [1; Chapter V. Proposition 1.1, page 122-123], we have

Proposition 2. (Reverse Hölder inequalities) Let $\Omega$ be an open domain and let $f$ and $g$ be positive functions. Suppose

$$
f_{B_{R}\left(x_{0}\right)} g^{q} d x \leq b\left(f_{B_{2 R}\left(x_{0}\right)} g d x\right)^{q}+f_{B_{2 R}\left(x_{0}\right)} f^{q} d x+\theta f_{B_{2 R}\left(x_{0}\right)} g^{q} d x
$$

for each $x_{0} \in \Omega$ and each $R<\frac{1}{2}$ dist $\left(x_{0}, \partial \Omega\right) \wedge R_{0}$, where $R_{0}, b, \theta$ are constants with $b>1, R_{0}>0,0 \leq \theta<1$. Then $g \in L_{l o c}^{p}(\Omega)$ for $p \in[q, q+\varepsilon)$ and

$$
\left(f_{B_{R}\left(x_{0}\right)} g^{p} d x\right)^{1 / p} \leq c\left(f_{B_{2 R}\left(x_{0}\right)} g^{q} d x\right)^{1 / q}+c\left(f_{B_{2 R}\left(x_{0}\right)} f^{p} d x\right)^{1 / p}
$$

for $B_{2 R} \subset \Omega, R<R_{0}$, where $c$ and $\varepsilon$ are positive constants depending on $b, \theta, n$. 
THEOREM 3. (L $L^{p}$-estimates) Let $(u, H)$ be a weak solution of (1.1)-(1.2) with $u \in W^{1, q}(\Omega, \mathbb{R}), H \in W^{1,2}\left(\Omega, \mathbb{R}^{n}\right)$ and $d^{*} H(x)=0$ for a.e. $x \in \Omega$. Then there exists a small positive constant $\varepsilon$ such that $H \in W_{\text {loc }}^{1, p}\left(\Omega, \mathbb{R}^{n}\right)$ for some $p \in(2,2+\varepsilon)$. More precisely,

$$
\left(f_{B_{R}\left(x_{0}\right)}|\nabla H|^{p} d x\right)^{1 / p} \leq c\left(f_{B_{2 R}\left(x_{0}\right)}|\nabla H|^{2} d x\right)^{1 / 2}
$$

for all $x_{0} \in \Omega$ and all $R$ with $2 R<R_{0}$ with $B_{R_{0}}\left(x_{0}\right) \subset \Omega$ for some $R_{0}>0$. Moreover $u \in W_{\text {loc }}^{1, q_{1}}$ with $q_{1}=\frac{n p}{(2 n-p)}>\frac{n}{n-1}$ where $p>2$ is fixed above.

Proof. By the Sobolev-Poincare inequality, we have

$$
\int_{B_{R}}\left|H-(H)_{x_{0}, R}\right|^{2} d x \leq C R^{2+\left(1-\frac{2}{q_{2}}\right) n}\left(\int_{B_{R}}|\nabla H|^{q_{2}} d x\right)^{2 / q_{2}}
$$

for $q_{2}=\frac{2 n}{n+2}<2$.

Letting $\rho=R / 2$ in Lemma 1 , we have

$$
\left(f_{B_{R / 2}\left(x_{0}\right)}|\nabla H|^{2} d x\right)^{1 / 2} \leq C\left(f_{B_{R}\left(x_{0}\right)}|\nabla H|^{q_{2}} d x\right)^{1 / q_{2}} .
$$

Applying Proposition 2, there exists a $p>2$ such that $H \in W^{1, p}\left(\Omega ; R^{n}\right)$ and $(2.2)$ holds. Applying the standard $L^{p}$-theory for equation (1.2), we get $u \in W_{l o c}^{2, p / 2}(\Omega ; \mathbb{R})$. By Sobolev's inequality again, we have $u \in W^{1, \frac{n p}{(2 n-p)}}$.

3. Proof of Theorem A. In this section, we give a proof of Theorem A.

Let $\Omega(x, \rho)=\Omega \cap B_{\rho}(x)$ and let $p \geq 1$ and $\lambda \geq 0$. At first, let us define the Morrey space $L^{p, \lambda}(\Omega)$ in the following

Definition A. (Morrey spaces) We say that $u$ belongs to $L^{p, \lambda}(\Omega)$ if $u \in L^{p}(\Omega)$ satisfies

$$
\|u\|_{L^{p, \lambda}(\Omega)}=\left\{\sup _{x_{0} \in \Omega, 0<\rho<\operatorname{diam} \Omega} \rho^{-\lambda} \int_{\Omega\left(x_{0}, \rho\right)}|u|^{p} d x\right\}^{1 / p}<+\infty
$$

and the Campanato space $\mathcal{L}^{p, \lambda}(\Omega)$

Definition B. (Campanato space) We say that u belongs to $\mathcal{L}^{p, \lambda}(\Omega)$ if $u \in L^{p}(\Omega)$ satisfies

$$
[u]_{p, \lambda}=\left\{\sup _{x_{0} \in \Omega, 0<\rho<\operatorname{diam} \Omega} \rho^{-\lambda} \int_{\Omega\left(x_{0}, \rho\right)}\left|u-u_{x_{0}, \rho}\right|^{p} d x\right\}^{1 / p}<+\infty,
$$

where $u_{x_{0}, \rho}=\frac{1}{|\Omega(x, \rho)|} \int_{\Omega(x, \rho)} u(x) d x$.

Let us recall some results about Morrey and Campanato spaces from [1] and [2]. If there exists a constant $A$ such that $|\Omega(x, \rho)| \geq A \rho^{n}$ for all $\Omega(x, \rho)$, the Campanato space $\mathcal{L}^{p, \lambda}(\Omega)$ is isomorphic to the Morrey space $L^{p, \lambda}(\Omega)$ when $0 \leq \lambda<n$, and moreover, when $n<\lambda \leq n+p, \mathcal{L}^{p, \lambda}(\Omega)$ is isomorphic to the Hölder space $C^{0, \alpha}$ with $\alpha=\frac{\lambda-n}{p}$. 
LEMma 4. Let $(u, H)$ be a weak solution to (1.1)-(1.2). Then $u$ is also a weak solution to the following equation

$$
\triangle u=d^{*}[\sigma(u)\langle d H, H\rangle],
$$

where

$$
\langle d H, H\rangle:=\sum_{i, j=1}^{n} F^{i j} H^{j} d x_{i} .
$$

Proof. Taking $\phi H$ as a test function in (1.1), we obtain

$$
\int_{\Omega}\langle\sigma(u) d H, d(\phi H)\rangle d x=0
$$

where $\phi$ is a function with $\phi \in C_{0}^{2}(\Omega ; \mathbb{R})$. Then by the definition in Section 1 , we get

$$
\begin{aligned}
\int_{\Omega} \phi \sigma(u)|d H|^{2} d x & =-\int_{\Omega}\langle\sigma(u) d H, d \phi \wedge H\rangle d x \\
& =-\int_{\Omega} \sigma(u)\left\langle\sum_{i, j} F^{i j} H^{j} d x_{i}, \sum_{m} \frac{\partial \phi}{\partial x_{m}} d x_{m}\right\rangle d x \\
& =-\int_{\Omega} \phi d^{*}[\sigma(u)\langle d H, H\rangle] d x
\end{aligned}
$$

for all $\phi \in C_{0}^{2}(\Omega ; \mathbb{R})$, where $\langle d H, H\rangle$ is defined in (3.2). This proves our claim.

Now we prove partial regularity of the weak solutions $(u, H)$ to the system (1.1)$(1.2)$.

Proof of Theorem $A$. Under the gauge condition $d^{*} H=0$, we know from the Hodge theory that

$$
-\triangle H=d^{*} d H+d d^{*} H=d^{*} d H
$$

Let $x_{0} \in \Omega$ with $B_{R_{0}}\left(x_{0}\right) \subset \subset \Omega$ for some $R_{0}>0$. Let a 1 -form $H_{1} \in W^{1,2}\left(B_{R}\left(x_{0}\right)\right)$ be a weak solution of the following Dirichlet problem

$$
\begin{gathered}
\sigma\left(u_{x_{0}, R}\right) \triangle H_{1}=0, \forall x \in B_{R}\left(x_{0}\right), \\
H_{1}-H \in W_{0}^{1,2}\left(B_{R}\left(x_{0}\right), \mathbb{R}^{n}\right) .
\end{gathered}
$$

Then for all $\rho<R \leq R_{0}$, we have

$$
\int_{B_{\rho}\left(x_{0}\right)}\left|\nabla H_{1}\right|^{2} d x \leq C\left(\frac{\rho}{R}\right)^{n} \int_{B_{R}\left(x_{0}\right)}\left|\nabla H_{1}\right|^{2} d x
$$

and therefore for all $\rho<R \leq R_{0}$ with some $R_{0}>0$

$$
\int_{B_{\rho}\left(x_{0}\right)}|\nabla H|^{2} d x \leq C\left(\frac{\rho}{R}\right)^{n} \int_{B_{R}\left(x_{0}\right)}|\nabla H|^{2} d x+C \int_{B_{R}\left(x_{0}\right)}\left|\nabla\left(H-H_{1}\right)\right|^{2} d x .
$$

Let $W=H-H_{1}$. Using equations (1.1) and (3.3), $W$ is the weak solution of the following

$$
\sigma\left(u_{x_{0}, R}\right) \triangle W=d^{*}\left\{\left[\sigma(u)-\sigma\left(u_{x_{0}, R}\right)\right] d H\right\}
$$


with boundary condition $W=0$ on $\partial B_{R}\left(x_{0}\right)$. Using $W$ as a test function in the above equation, we get

$$
\sigma\left(u_{x_{0}, R}\right) \int_{B_{R}}|\nabla W|^{2} d x=-\int_{B_{R}}\left\langle\left[\sigma(u)-\sigma\left(u_{x_{0}, R}\right)\right] d H, d W\right\rangle d x .
$$

By the assumption on $\sigma(u)$, there exists a non-negative, bounded function $\omega(t)$ increasing in $t$, concave, continuous with $\omega(0)=0$, such that for $u, v \in \mathbb{R}$,

$$
|\sigma(u)-\sigma(v)| \leq \omega\left(|u-v|^{q_{1}}\right),
$$

where $q_{1}=\frac{n p}{2 n-p}$ and $p$ is a fixed exponent in $(2,2+\varepsilon)$ from Theorem 3 . Hence we get from (3.5)-(3.6)

$$
\int_{B_{R}\left(x_{0}\right)}|\nabla W|^{2} d x \leq C \int_{B_{R}\left(x_{0}\right)} \omega^{2}\left(\left|u-u_{x_{0}, R}\right|^{q_{1}}\right)|\nabla H|^{2} d x .
$$

By the Sobolev-Poincare inequality, we obtain

$$
\int_{B_{R}}\left|u-u_{x_{0}, R}\right|^{q_{1}} d x \leq C R^{q_{1}} \int_{B_{R}}|\nabla u|^{q_{1}} d x .
$$

Using the $L^{p}$-estimate (Theorem 3 ) and the boundedness and concavity of $\omega$, we have

$$
\begin{aligned}
& \int_{B_{R}\left(x_{0}\right)} \omega^{2}\left(\left|u-u_{x_{0}, R}\right|^{q_{1}}\right)|\nabla H|^{2} d x \\
& \leq C\left(\int_{B_{R}\left(x_{0}\right)}|\nabla H|^{p} d x\right)^{2 / p}\left(\int_{B_{R}\left(x_{0}\right)} \omega^{\frac{2 p}{p-2}}\left(\left|u-u_{x_{0}, R}\right|^{q_{1}}\right) d x\right)^{\frac{p-2}{p}} \\
& \leq C\left(\int_{B_{2 R}\left(x_{0}\right)}|\nabla H|^{2} d x\right)\left(\left|B_{R}\left(x_{0}\right)\right|^{-1} \int_{B_{R}\left(x_{0}\right)} \omega\left(\left|u-u_{x_{0}, R}\right|^{q_{1}}\right) d x\right)^{\frac{p-2}{p}} \\
& \leq C \omega^{\frac{p-2}{p}}\left(C R^{q_{1}-n} \int_{B_{R}\left(x_{0}\right)}|\nabla u|^{q_{1}} d x\right)\left(\int_{B_{2 R}}|\nabla H|^{2} d x\right),
\end{aligned}
$$

where last inequality comes from the concavity of $\omega$ using the Jensen inequality and the Poincare inequality.

Therefore for all $\rho<R<2 R \leq R_{0}$ we have

$$
\begin{aligned}
\int_{B_{\rho}\left(x_{0}\right)}|\nabla H|^{2} d x & \leq C\left(\frac{\rho}{R}\right)^{n} \int_{B_{2 R}\left(x_{0}\right)}|\nabla H|^{2} d x+ \\
& +C \omega^{\frac{p-2}{p}}\left(C R^{q_{1}-n} \int_{B_{2 R}\left(x_{0}\right)}|\nabla u|^{q_{1}} d x\right) \int_{B_{2 R}\left(x_{0}\right)}|\nabla H|^{2} d x
\end{aligned}
$$

By Theorem 3, $u$ belongs to $W^{2, p / 2}(\Omega)$. Let $v \in W^{2, p / 2}\left(B_{R}\left(x_{0}\right)\right)$ be a weak solution of the following Dirichlet problem:

$$
\begin{gathered}
-\triangle v=0, \quad \text { in } B_{R}\left(x_{0}\right), \\
\left.v\right|_{\partial B_{R}}=\left.u\right|_{\partial B_{R}}, \quad x \in \partial B_{R}\left(x_{0}\right) .
\end{gathered}
$$


For the harmonic function $v$, it is easy to see that for $\rho \leq R<2 R \leq R_{0}$, we obtain

$$
\int_{B_{\rho}\left(x_{0}\right)}|\nabla v|^{q_{1}} d x \leq C\left(\frac{\rho}{R}\right)^{n} \int_{B_{R}\left(x_{0}\right)}|\nabla v|^{q_{1}} d x .
$$

Let $w=u-v$. Then $w \in W^{2, p / 2}\left(B_{R}\left(x_{0}\right) ; \mathbb{R}\right)$ satisfies

$$
\begin{gathered}
-\triangle w=\sigma(u)|d H|^{2}, \quad \text { in } B_{R}\left(x_{0}\right), \\
w=0 \quad \text { on } \partial B_{R}\left(x_{0}\right) .
\end{gathered}
$$

Then

$$
\int_{B_{\rho}\left(x_{0}\right)}|\nabla u|^{q_{1}} d x \leq C\left(\frac{\rho}{R}\right)^{n} \int_{B_{R}\left(x_{0}\right)}|\nabla u|^{q_{1}} d x+C \int_{B_{R}}|\nabla w|^{q_{1}} d x .
$$

We rescale

$$
\tilde{u}(x)=u\left(x_{0}+R x\right), \tilde{w}(x)=w\left(x_{0}+R x\right), \tilde{H}(x)=H\left(x_{0}+R x\right)=H^{i}\left(x_{0}+R x\right) d x_{i} .
$$

Then

$$
\begin{gathered}
-\triangle \tilde{w}=\sigma(u)|d \tilde{H}|^{2}, \quad \text { in } B_{1}, \\
\tilde{w}=0 ; \quad \text { on } \partial B_{1},
\end{gathered}
$$

where $B_{1}=B(0,1)$ is the unit ball in $\mathbb{R}^{n}$. Applying the standard elliptic $L^{p}$-theory (see [7]) to (3.8)-(3.9), we obtain

$$
\left(\frac{1}{\left|B_{1}\right|} \int_{B_{1}}\left|\nabla^{2} \tilde{w}\right|^{p / 2} d x\right)^{2 / p} \leq C\left(\frac{1}{\left|B_{1}\right|} \int_{B_{1}}|\nabla \tilde{H}|^{p} d x\right)^{2 / p}
$$

where $C$ is a constant independent of $R$.

Rescaling back, we have

$$
\left(\frac{1}{\left|B_{R}\left(x_{0}\right)\right|} \int_{B_{R}\left(x_{0}\right)}\left|\nabla^{2} w\right|^{p / 2} d x\right)^{2 / p} \leq C\left(\frac{1}{\left|B_{R}\left(x_{0}\right)\right|} \int_{B_{R}\left(x_{0}\right)}|\nabla H|^{p} d x\right)^{2 / p},
$$

where $C$ is a constant independent of $R$. By the Sobolev inequality and using $L^{p}$ estimates, we see

$$
\begin{aligned}
\left(f_{B_{R}\left(x_{0}\right)}|\nabla w|^{q_{1}} d x\right)^{\frac{1}{q_{1}}} & \leq C R\left(f_{B_{R}\left(x_{0}\right)}\left|\nabla^{2} w\right|^{p / 2} d x\right)^{2 / p} \\
& \leq C R^{1-n} \int_{B_{2 R}\left(x_{0}\right)}|\nabla H|^{2} d x
\end{aligned}
$$

Therefore for all $\rho<R<2 R \leq R_{0}$, we have

$$
\begin{aligned}
\int_{B_{\rho}\left(x_{0}\right)}|\nabla u|^{q_{1}} d x \leq & C\left(\frac{\rho}{R}\right)^{n} \int_{B_{2 R}\left(x_{0}\right)}|\nabla u|^{q_{1}} d x \\
& +C R^{n+q_{1}(1-n)}\left(\int_{B_{2 R}\left(x_{0}\right)}|\nabla H|^{2} d x\right)^{q_{1}} .
\end{aligned}
$$


For any $x_{0} \in \Omega$ and $r$ with $R_{0} \geq r>0$, we denote

$$
\Phi\left(x_{0}, r\right)=r^{2-n} \int_{B_{r}\left(x_{0}\right)}|\nabla H|^{2} d x, \quad \xi\left(x_{0}, r\right)=r^{q_{1}-n} \int_{B_{r}\left(x_{0}\right)}|\nabla u|^{q_{1}} d x,
$$

Note that (3.7) and (3.10) also hold for $R<\rho<2 R \leq R_{0}$. Then for all $\tau<1$, we have

$$
\Phi\left(x_{0}, \tau R\right) \leq C_{1}\left[1+\omega^{\frac{p-2}{p}}\left(C_{2} \xi\left(x_{0}, R\right)\right) \tau^{-n}\right] \tau^{2} \Phi\left(x_{0}, R\right)
$$

and

$$
\xi\left(x_{0}, \tau R\right) \leq C_{1} \tau^{q_{1}} \xi\left(x_{0}, R\right)+\tau^{q_{1}-n} \Phi^{q_{1}}\left(x_{0}, R\right)
$$

by using $R$ instead of $2 R$ in (3.7) and (3.10). For any $\alpha<1$, choose $\tau<1$ such that

$$
2 C_{1} \tau^{q_{1} \alpha}=1
$$

There exists a small constant $\varepsilon_{0}>0$ such that if

$$
\xi\left(x_{0}, R\right)+\Phi\left(x_{0}, R\right)<\varepsilon_{0}
$$

for some $R<R_{0}$, then we have

$$
\Phi^{q_{1}-1}\left(x_{0}, R\right)<\tau^{n}, \quad \omega^{\frac{p-2}{p}}\left(C_{2} \xi\left(x_{0}, R\right)\right)<\tau^{n}
$$

provided that $R$ is less than some $R_{0}$. Hence

$$
\xi\left(x_{0}, \tau R\right)+\Phi\left(x_{0}, \tau R\right) \leq \tau^{2 \alpha}\left[\xi\left(x_{0}, R\right)+\Phi\left(x_{0}, R\right)\right] .
$$

Therefore by iteration we obtain

$$
\xi\left(x_{0}, \tau^{k} R\right)+\Phi\left(x_{0}, \tau^{k} R\right) \leq \tau^{2 k \alpha}\left[\xi\left(x_{0}, R\right)+\Phi\left(x_{0}, R\right)\right]<\varepsilon_{0}
$$

In conclusion, if $\xi\left(x_{0}, R\right)+\Phi\left(x_{0}, 2 R\right)<\varepsilon_{0}$ for some $R<R_{0}$, then

$$
\xi\left(x_{0}, \tau^{k} R\right)+\Phi\left(x_{0}, \tau^{k} R\right) \leq \tau^{2 k \alpha} \varepsilon_{0} .
$$

Hence for any $\rho<R_{0}$, we have

$$
\xi\left(x_{0}, \rho\right)+\Phi\left(x_{0}, \rho\right) \leq C\left(\frac{\rho}{R}\right)^{2 \alpha},
$$

where $C$ is a constant independent of $\rho$ and $R$.

Note that $\xi\left(x_{0}, R\right)$ and $\Phi\left(x_{0}, R\right)$ are continuous functions of $x_{0}$. There exits an open $\Omega_{0} \subset \Omega$ such that $u$ and $H$ are in $C_{\text {loc }}^{0, \alpha}\left(\Omega_{0}\right)$ for every $\alpha<1$. Moreover, $\Omega \backslash \Omega_{0} \subset \Sigma_{1} \cup \Sigma_{2}$, where

$$
\begin{aligned}
& \Sigma_{1}=\left\{x \in \Omega: \liminf _{R \rightarrow 0^{+}} R^{2-n} \int_{B_{R}(x)}|\nabla H|^{2} d x>0\right\}, \\
& \Sigma_{2}=\left\{x \in \Omega: \liminf _{R \rightarrow 0^{+}} R^{q_{1}-n} \int_{B_{R}(x)}|\nabla u|^{q_{1}} d x>0\right\} .
\end{aligned}
$$

Moreover, since $H \in W^{1,2}\left(\Omega, \mathbb{R}^{n}\right)$ and $u \in W_{l o c}^{1, q_{1}}\left(\Omega, R^{n}\right)$ with $q_{1}=\frac{n p}{2 n-p}$ for some $p>2$, we have

$$
\mathcal{H}^{n-q_{1}}\left(\Omega \backslash \Omega_{0}\right)=0
$$

where $\mathcal{H}^{n-q_{1}}$ denote $\left(n-q_{1}\right)$-Hausdorff measure. 
Next we prove $C^{1, \alpha}$-regularity inside $\Omega_{0}$. We assume that $x_{0} \in \Omega$ with $B_{2 R}\left(x_{0}\right) \subset$ $\Omega_{0}$. From the above results, we know that $u$ and $H$ are $C^{0, \alpha}\left(\Omega_{0}\right)$ for every $\alpha<1$ and

$$
R^{q_{1}-n} \int_{B_{R}\left(x_{0}\right)}|\nabla u|^{q_{1}} d x \leq C R^{2 \alpha}, \quad R^{2-n} \int_{B_{R}\left(x_{0}\right)}|\nabla H|^{2} d x \leq C R^{2 \alpha},
$$

where $C$ is a constant independent of $R$. Note that $H_{1}$ is the solution to equations (3.3)-(3.4). For any $\rho$ and $R$ with $\rho<R \leq R_{0}$, we have

$$
\int_{B_{\rho}\left(x_{0}\right)}\left|\nabla H_{1}-\left(\nabla H_{1}\right)_{x_{0}, \rho}\right|^{2} d x \leq C\left(\frac{\rho}{R}\right)^{n+2} \int_{B_{R}\left(x_{0}\right)}\left|\nabla H_{1}-\left(\nabla H_{1}\right)_{x_{0}, \rho}\right|^{2} d x .
$$

Repeating the same proof as before (3.7), we get

$$
\int_{B_{\rho}\left(x_{0}\right)}|\nabla W|^{2} d x \leq C \omega^{\frac{p-2}{p}}\left(C R^{q_{1}-n} \int_{B_{R}\left(x_{0}\right)}|\nabla u|^{2} d x\right) \int_{B_{R}\left(x_{0}\right)}|\nabla H|^{2} d x
$$

for some $p>2$.

Since $\omega$ is uniformly Hölder continuous, there exist constants $\beta$ and $C$ with $0<$ $\beta<1$ such that $\omega(t) \leq C t^{\beta}$. Therefore

$$
\begin{aligned}
\int_{B_{\rho}\left(x_{0}\right)}\left|\nabla H-(\nabla H)_{x_{0}, \rho}\right|^{2} d x \leq & C\left(\frac{\rho}{R}\right)^{n+2} \int_{B_{2 R}\left(x_{0}\right)}\left|\nabla H-(\nabla H)_{x_{0}, R}\right|^{2} d x \\
& +C R^{n-2+\alpha\left[2+\beta \frac{p-2}{p}\right]}
\end{aligned}
$$

where $\alpha\left[2+\beta \frac{p-2}{p}\right]>2$ by letting $\alpha$ be closing to 1 . Then the standard procedure yields that $\nabla H$ is $C^{0, \gamma}$ for some $0<\gamma<1$. By applying standard PDE theory to equation (1.2), it is easy to see that $\nabla u$ is also locally in $C_{l o c}^{0, \gamma_{1}}\left(\Omega_{0}\right)$ for some $\gamma_{1}>0$. This proves our claim.

4. Partial regularity for the parabolic system. In this section, we prove the partial regularity of the weak solutions to system (1.5)-(1.6).

Denote $Q_{T}=\Omega \times(0, T)$ and let $z=(x, t)$ for $x \in \Omega$ and $t \in(0, T)$. We recall some definitions from [9]. $L_{p, r}\left(Q_{T}\right)$ is the Banach space consisting of all measurable functions on $Q_{T}$ with a finite norm

$$
\|u\|_{p, r, Q_{T}}=\left(\int_{0}^{T}\left(\int_{\Omega}|u(x, t)|^{p} d x\right)^{r / p} d t\right)^{1 / r} .
$$

We denote $\|u\|_{p, Q_{T}}=\|u\|_{p, p, Q_{T}}$. The space $V_{p}^{1,0}\left(Q_{T}\right)$ is the completion of $C^{1}\left(Q_{T}\right)$ with respect to the norm

$$
|u|_{p ; Q_{T}}=\left\{\int_{Q_{T}}\left(|u|^{p}+|\nabla u|^{p}\right) d z\right\}^{1 / p} .
$$

The space $W_{p}^{2,1}\left(Q_{T}\right)$ with $p \geq 1$ is the Banach space consisting of the elements of $L_{p}\left(Q_{T}\right)$ having generalized derivatives of the form $D_{t}^{r} D_{x}^{s}$ with any $r$ and $s$ satisfying the inequality $2 r+s \leq 2$. The norm is defined by

$$
\|u\|_{q, Q_{T}}^{(2)}=\sum_{j=0}^{2}\langle\langle u\rangle\rangle_{q, Q_{T}}^{(j)}
$$


with

$$
\langle\langle u\rangle\rangle_{p, Q_{T}}^{(j)}=\sum_{2 r+s=j}\left\|D_{t}^{r} D_{x}^{s} u\right\|_{q, Q_{T}} .
$$

We say that a pair $(u, H)$ is a weak solution to equations (1.6)-(1.7) if $u \in$ $V_{q}^{1,0}\left(Q_{T}\right)$ for some $q \in\left(1, \frac{n}{n-1}\right)$ and $H^{i} \in V_{2}^{1,0}\left(Q_{T} ; \mathbb{R}^{n}\right)$, and the pair $(u, H)$ satisfies the following:

$$
\begin{gathered}
\int_{Q_{T}}\left[\left\langle H, \partial_{t} \phi\right\rangle+\langle\sigma(u) d H, d \phi\rangle\right] d z=0, \\
\int_{Q_{T}}\left[-u \psi_{t}+\nabla u \cdot \nabla \psi\right] d z=\int_{Q_{T}} \sigma(u)|d H|^{2} \psi d z
\end{gathered}
$$

for all $\phi:=\sum_{i=1}^{n} \phi^{i}(x, t) d x_{i}$ for $i=1, \ldots, n$ with $d^{*} H=0$ in $Q_{T}$ in the weak sense, where $\phi^{i}(x, t) \in C_{0}^{2}\left(Q_{T} ; \mathbb{R}\right)$ and $\psi(x, t) \in C_{0}^{2}\left(Q_{T} ; \mathbb{R}\right)$. The existence of weak solutions of (4.1)-(4.2) with $d^{*} H=0$ in $Q_{T}$ was obtained by Yin in [13] and [14].

For any $R>0$, denote $Q_{R}\left(z_{0}\right)=B_{R}\left(x_{0}\right) \times\left(t_{0}-R^{2}, t_{0}+R^{2}\right)$ with $z_{0}=\left(x_{0}, t_{0}\right)$. We denote for any function $u(x, t)$

$$
u_{z_{0}, R}=f_{Q_{R}\left(z_{0}\right)} u(z) d z
$$

Next, we prove partial regularity of weak solutions to the system (4.1)-(4.2) by modifying the method for elliptic case of Sections 2-3. The first step towards the proof of Theorem B is to establish a Caccioppoli's inequality and $L^{p}$-estimates for weak solutions to the parabolic system (4.1)-(4.2) by applying the proof of [3] and [6]. More precisely, we have

Lemma 7. (Caccioppoli's inequality for parabolic problems) Assume that $(u, H)$ is a weak solution of (4.1)-(4.2) with the assumptions of Theorem $B$. Then there exists a constant $C$ such that for any $x_{0} \in Q_{T}$ and any $R$ with $2 R \leq R_{0}$ with $Q_{R_{0}}\left(z_{0}\right) \subset Q_{T}$ for some $R_{0}>0$,

$$
\int_{Q_{R}\left(z_{0}\right)}|\nabla H|^{2} d z \leq \frac{C}{R^{2}} \int_{Q_{2 R}\left(z_{0}\right)}\left|H-\tilde{H}_{x_{0}, 2 R}(t)\right|^{2} d z .
$$

Proof. Let $z_{0}=\left(x_{0}, t_{0}\right) \in Q_{T}$. Let $\xi(x)$ be a function in $C_{0}^{\infty}\left(B_{2}\left(x_{0}\right)\right)$ such that $0 \leq \xi \leq 1, \xi=1$ in $B_{1}\left(x_{0}\right)$ and $|\nabla \xi| \leq 2$. We also denote by $\xi_{R}$ the function $\xi_{2 R}(x)=\xi\left(\frac{x}{R}\right)$. As in [6], for a function $H^{i}(x, t)$, we define the weighted means of $H^{i}(x, t)$ in $B_{2 R}\left(x_{0}\right)$ as

$$
\tilde{H}_{x_{0}, 2 R}^{i}(t)=\frac{\int_{B_{2 R}\left(x_{0}\right)} H^{i}(x, t) \xi_{2 R}^{2} d x}{\int_{B_{2 R}\left(x_{0}\right)} \xi_{2 R}^{2}(x) d x}
$$

Then we define

$$
\tilde{H}_{x_{0}, 2 R}(t)=\sum_{i} \tilde{H}_{x_{0}, 2 R}^{i}(t) d x_{i}
$$


Let $\tau \in C^{\infty}(\mathbb{R}, \mathbb{R})$ be a function only in $t$ and satisfy $0 \leq \tau \leq 1, \tau \equiv 1$ on $\left[t_{0}-R^{2}, t_{0}\right]$, $\tau \equiv 0$ on $t<t_{0}-(2 R)^{2}$. By the above choice, we note

$$
\int_{t_{0}-4 R^{2}}^{t_{0}}\left[\int_{B_{2 R}\left(x_{0}\right)}\left(H^{i}-H_{2 R}^{i}(t)\right) \xi^{2} d x\right] \partial_{t} \tilde{H}_{2 R}^{i}(t) \tau^{2} d t=0 .
$$

Let $\mathbb{I}_{\left(-\infty, t_{0}\right)}$ be the characteristic function of the interval $\left(-\infty, t_{0}\right)$. Testing $\phi=$ $\left(H-\tilde{H}_{2 R}(t)\right) \xi_{2 R}^{2} \tau^{2} \mathbb{I}_{\left(-\infty, t_{0}\right)}$ and noting (4.3), we have

$$
\begin{aligned}
& \int_{B\left(x_{0}, 2 R\right) \times\left\{t_{0}\right\}}\left|H-\tilde{H}_{x_{0}, 2 R}(t)\right|^{2} \xi^{2} \tau^{2} d x+\int_{Q_{2 R}\left(z_{0}\right)} \sigma(u)|d H|^{2} \xi^{2} \tau^{2} d z \\
& \leq 2 \int_{Q_{2 R}\left(z_{0}\right)}\left|H-\tilde{H}_{x_{0}, 2 R}(t)\right|^{2} \xi^{2} \tau \partial_{t} \tau d z \\
& \quad-2 \int_{Q_{2 R}\left(z_{0}\right)} \sigma(u)\left\langle d H, \xi d \xi \wedge\left(H-\tilde{H}_{x_{0}, 2 R}(t)\right)\right\rangle \tau^{2} d z .
\end{aligned}
$$

It follows from (4.4) that

$$
\int_{Q_{R}}|d H|^{2} \xi^{2} \tau^{2} d z \leq \frac{C}{R^{2}} \int_{Q_{2 R}\left(z_{0}\right)}\left|H-\tilde{H}_{x_{0}, 2 R}(t)\right|^{2} d z .
$$

A similar argument to Lemma 1 yields

$$
\begin{aligned}
& \int_{t_{0}-R^{2}}^{t_{0}} \int_{B_{2 R}\left(x_{0}\right)}|d H|^{2} \xi^{2} d x \tau^{2} d t \\
& =\int_{t_{0}-R^{2}}^{t_{0}}\left(\int_{B_{2 R}\left(x_{0}\right)}|\nabla H|^{2} \xi^{2} d x-\sum_{i, j=1}^{n} \int_{B_{2 R}\left(x_{0}\right)} \frac{\partial H^{i}}{\partial x_{j}} \frac{\partial H^{j}}{\partial x_{i}} \xi^{2} d x\right) \tau^{2} d t \\
& =\int_{t_{0}-R^{2}}^{t_{0}} \int_{B_{2 R}\left(x_{0}\right)}\left(|\nabla H|^{2} \xi^{2}+2 \sum_{i, j=1}^{n} \frac{\partial H^{i}}{\partial x_{j}} \xi \frac{\partial \xi}{\partial x_{i}}\left[H^{j}-\tilde{H}_{x_{0}, 2 R}^{j}(t)\right]\right) d x \tau^{2} d t \\
& +\int_{t_{0}-R^{2}}^{t_{0}} \int_{B_{2 R}\left(x_{0}\right)} \sum_{i, j=1}^{n} \xi^{2}\left[H^{j}-\tilde{H}_{x_{0}, 2 R}^{j}(t)\right] \frac{\partial^{2} H^{i}}{\partial x_{j} \partial x_{i}} d x \tau^{2} d t .
\end{aligned}
$$

By using $d^{*} H=0$, the last term in above identity is zero. This proves our claim.

We have the following $L^{p}$-estimate:

Lemma 8. Let $(u, H)$ be a weak solution to the system (4.1)-(4.2) with the assumptions of Theorem B. Then there exists an exponent $p>2$ such that $\nabla H \in$ $L_{\text {loc }}^{p}\left(Q_{T}\right)$; moreover for all $Q_{R}\left(z_{0}\right) \subset Q_{4 R}\left(z_{0}\right) \subset Q_{T}$ we have

$$
f_{Q_{R}\left(z_{0}\right)}|\nabla H|^{p} d z \leq C\left(f_{Q_{4 R}\left(z_{0}\right)}|\nabla H|^{2} d z\right)^{\frac{p}{2}}
$$

and $u \in W_{p / 2 ; l o c}^{2,1}\left(Q_{T}\right)$.

For the proof of Lemma 8, the same proof as in [5] gives the desired $L^{p}$-estimate for $H$ by using the reverse Hölder inequality as in Proposition 3. The fact $u \in$ 
$W_{p / 2 ; l o c}^{2,1}\left(Q_{T}\right)$ follows from Theorem 9.1 of Chapter IV of [10; pages 341-2].

By a slight modification of arguments in [12] (for the details, see [14]), we have

Lemma 9. Let $(u, H)$ be a weak solution to the system (4.1)-(4.2) with the assumptions of Theorem $B$. Then for all $Q_{R}\left(z_{0}\right) \subset Q_{2 R}\left(z_{0}\right) \subset Q_{T}$, we have

$$
\int_{Q_{R}\left(z_{0}\right)}\left|H-H_{R, z_{0}}\right|^{2} d z \leq C R^{2} \int_{Q_{2 R}\left(z_{0}\right)}|\nabla H|^{2} d z .
$$

Now we complete the proof of Theorem B.

Proof of Theorem B. For any $z_{0} \in Q_{T}$, choose $R_{0}$ with $Q_{R_{0}}\left(z_{0}\right) \subset Q_{T}$. Let $S_{R}\left(z_{0}\right)$ be the parabolic boundary of $Q_{R}\left(z_{0}\right)$ defined by

$$
S_{R}=B_{R}\left(x_{0}, t_{0}-R^{2}\right) \cup\left[\partial B_{R}\left(x_{0}\right) \times\left(t_{0}-R^{2}, t_{0}+R^{2}\right)\right] .
$$

Let a 1 -form $H_{1} \in V_{2}^{1,0}\left(Q_{R}\left(z_{0}\right)\right)$ be the weak solution of the following parabolic problem:

$$
\begin{gathered}
\partial_{t} H_{1}=\sigma\left(u_{z_{0}, R}\right) \triangle H_{1}, \quad \text { in } Q_{R}\left(z_{0}\right), \\
\left.H_{1}\right|_{S_{R}\left(z_{0}\right)}=\left.H\right|_{S_{R}\left(z_{0}\right)}, \quad \text { on } S_{R}\left(z_{0}\right) .
\end{gathered}
$$

For all $\rho<R \leq R_{0}$, we have

$$
\int_{Q_{\rho}\left(z_{0}\right)}|\nabla H|^{2} d z \leq C\left(\frac{\rho}{R}\right)^{n+2} \int_{Q_{R}\left(z_{0}\right)}|\nabla H|^{2} d z+C \int_{Q_{R}\left(x_{0}\right)}|\nabla W|^{2} d z
$$

with $W=H-H_{1}$. By a similar proof as in Section 3, we have

$$
\int_{Q_{R}\left(z_{0}\right)}|\nabla W|^{2} d z \leq C \omega^{\frac{p-2}{p}}\left(C R^{-n} \int_{Q_{4 R}\left(z_{0}\right)}\left|u-u_{z_{0}, R}\right|^{p / 2} d z\right) \int_{Q_{2 R}\left(z_{0}\right)}|\nabla H|^{2} d z .
$$

Let $v \in W_{p / 2}^{2,1}\left(Q_{R}\left(z_{0}\right)\right)$ be a weak solution of

$$
\begin{aligned}
\partial_{t} v & =\Delta v, \quad \text { in } Q_{R}\left(z_{0}\right), \\
\left.v\right|_{S_{R}\left(z_{0}\right)} & =\left.u\right|_{S_{R}\left(z_{0}\right)}, \quad \text { on } S_{R}\left(z_{0}\right) .
\end{aligned}
$$

Then for all $\rho<R \leq R_{0}$, we have

$$
\int_{Q_{\rho}\left(z_{0}\right)}\left|\partial_{t} u\right|^{p / 2} d z \leq C\left(\frac{\rho}{R}\right)^{n+2} \int_{Q_{R}\left(z_{0}\right)}\left|\partial_{t} u\right|^{p / 2} d z+C \int_{Q_{R}\left(z_{0}\right)}\left|\partial_{t} w\right|^{p / 2} d z
$$

and

$$
\int_{Q_{\rho}\left(z_{0}\right)}|\nabla u|^{p / 2} d z \leq C\left(\frac{\rho}{R}\right)^{n+2} \int_{Q_{R}\left(z_{0}\right)}|\nabla u|^{p / 2} d z+C \int_{Q_{R}\left(z_{0}\right)}|\nabla w|^{p / 2} d z,
$$

where $w=u-v$ satisfies

$$
\begin{gathered}
\partial_{t} w=\Delta w+\sigma(u)|d H|^{2}, \quad \text { in } Q_{R}\left(z_{0}\right), \\
w=0 \quad \text { on } S_{R}\left(z_{0}\right) .
\end{gathered}
$$


Since $|\nabla H|^{2}$ is locally in $L^{p / 2, p / 2}\left(Q_{T}\right)$, we have from Theorem 9.1 of [10; Chapter IV] and Lemma 8 that

$$
\begin{aligned}
\int_{Q_{R}\left(z_{0}\right)}\left(\left|\nabla^{2} w\right|^{p / 2}+\left|\partial_{t} u\right|^{p / 2}\right) d z & \leq C \int_{Q_{R}\left(z_{0}\right)}|\nabla H|^{p} d z \\
& \leq C R^{n+2}\left(f_{Q_{4 R}\left(z_{0}\right)}|\nabla H|^{2} d x\right)^{p / 2}
\end{aligned}
$$

By the Sobolev inequality and using $L^{p}$-estimates in (4.7), we know

$$
\begin{aligned}
\int_{Q_{R}\left(z_{0}\right)}|\nabla w|^{p / 2} d z & \leq C R^{p / 2} \int_{Q_{R}\left(z_{0}\right)}\left|\nabla^{2} w\right|^{p / 2} d z \\
& \leq C R^{n+2-\frac{p}{2}(n+1)}\left(\int_{Q_{4 R}\left(z_{0}\right)}|\nabla H|^{2} d x\right)^{p / 2}
\end{aligned}
$$

By a version of the Sobolev-Poincare inequality, we have

$$
\int_{Q_{R}\left(z_{0}\right)}\left|u-u_{z_{0}, R}\right|^{p / 2} d z \leq C\left[R^{p / 2} \int_{Q_{R}\left(z_{0}\right)}|\nabla u|^{p / 2} d z+R^{p} \int_{Q_{R}\left(z_{0}\right)}\left|\partial_{t} u\right|^{p / 2} d z\right] .
$$

For any $z_{0} \in Q_{T}$ and $r$ with $Q_{z_{0}, r} \subset Q_{T}$, we denote

$$
\begin{gathered}
\Phi\left(z_{0}, r\right)=r^{-n} \int_{Q_{r}\left(z_{0}\right)}|\nabla H|^{2} d z, \quad \xi\left(z_{0}, r\right)=r^{-n-2+p / 2} \int_{Q_{r}\left(z_{0}\right)}|\nabla u|^{p / 2} d z \\
\eta\left(z_{0}, r\right)=r^{-n-2+p} \int_{Q_{r}\left(z_{0}\right)}\left|\partial_{t} u\right|^{p / 2} \cdot d z .
\end{gathered}
$$

Then for all $\tau<1$, we have

$$
\begin{gathered}
\Phi\left(z_{0}, \tau R\right) \leq C_{1}\left[1+\omega^{\frac{p-2}{p}}\left(C_{2}\left[\xi\left(z_{0}, R\right)+\eta\left(z_{0}, R\right)\right]\right) \tau^{-(n+2)} \tau^{2} \Phi\left(x_{0}, R\right),\right. \\
\xi\left(z_{0}, \tau R\right) \leq C_{1} \tau^{2} \xi\left(x_{0}, R\right)+\tau^{\frac{p}{2}-(n+2)} \Phi^{\frac{p}{2}}\left(x_{0}, R\right)
\end{gathered}
$$

and

$$
\eta\left(z_{0}, \tau R\right) \leq C_{1} \tau^{p} \eta\left(z_{0}, R\right)+\tau^{p-(n+2)} \Phi^{\frac{p}{2}}\left(z_{0}, R\right) .
$$

If there exists a constant $\varepsilon_{0}$ such that $\Phi\left(z_{0}, r\right)+\xi\left(z_{0}, r\right)+\eta\left(z_{0}, r\right)<\varepsilon_{0}$ for some $r \leq R_{0}$, then a similar iteration step as in Section 3 yields

$$
\phi\left(z_{0}, \rho\right)+\xi\left(z_{0}, \rho\right)+\eta\left(z_{0}, \rho\right) \leq C \rho^{2 \alpha}
$$

for all $\alpha<1$ and $\rho \leq r \leq R_{0}$. Using the Sobolev inequality (4.8) and Lemma 9, we obtain through the Campanato space that $u(x, t)$ and $H(x, t)$ are Hölder continuous in $\alpha$ locally in $\tilde{Q}$ where $\tilde{Q}$ is an open subset of $Q_{T}$. A similar argument as in Section 3 yields that $u(x, t)$ and $H(x, t)$ are also in $C_{l o c}^{1, \gamma}(\tilde{Q})$ for some $\gamma<1$.

Since $u$ is in $W_{p / 2 ; l o c}^{2,1}\left(Q_{T}\right)$, we have $\nabla u \in L_{q_{3}, q_{3} ; l o c}\left(Q_{T}\right), q_{3}=\frac{(n+2) p}{n+2-2 p}$ by the parabolic type Sobolev inequality (see [10; Lemma 3.3, page 80 ]). Moreover, Hölder's 
inequality gives

$$
\xi\left(z_{0}, R\right) \leq\left(R^{q_{3}-n-2} \int_{Q_{z_{0}, R}}|\nabla u|^{q_{3}} d z\right)^{\frac{p}{2 q_{3}}}
$$

We have $Q_{T} \backslash \tilde{Q} \subset \Sigma_{1} \cup \Sigma_{2} \cup \Sigma_{3}$ where

$$
\begin{gathered}
\Sigma_{1}=\left\{z_{0} \in Q_{T}: \liminf _{R \rightarrow 0^{+}} R^{-n} \int_{Q_{z_{0}, R}}|\nabla H|^{2} d z>0\right\}, \\
\Sigma_{2}=\left\{z_{0} \in Q_{T}: \liminf _{R \rightarrow 0^{+}} R^{q_{3}-n-2} \int_{Q_{z_{0}, R}}|\nabla u|^{q_{3}} d z>0\right\},
\end{gathered}
$$

and

$$
\Sigma_{3}=\left\{z_{0} \in Q_{T}: \liminf _{R \rightarrow 0^{+}} R^{p-n-2} \int_{Q_{z_{0}, R}}\left|\partial_{t} u\right|^{p / 2} d z>0\right\} .
$$

Sine $\nabla H \in L_{2 ; l o c}\left(Q_{T}, \mathbb{R}^{n}\right)$ and $\partial_{t} u \in L_{p / 2}\left(Q_{T}, \mathbb{R}^{n}\right)$, we have

$$
\mathcal{H}^{n+2-q_{3}}\left(Q_{T} \backslash \tilde{Q}\right)=0
$$

where $\mathcal{H}^{n+2-q_{3}}$ denotes $\left(n+2-q_{3}\right)$-Hausdorff measure. This proves our claim.

\section{REFERENCES}

[1] M. Giaquinta, Mutilple integrals in the calculus of variations and nonlinear elliptic systems, Princeton Uviversity Press, 1983.

[2] M. Giaquinta, Mutilple integrals in the calculus of variations and nonlinear elliptic systems, Birkhäuser, Basel, 1993.

[3] M. Giaquinta and E. Giusti, Partial regularity for the solutions to nonlinear parabolic systems, Ann. Mat. Pura. Appl., 47 (1973), pp. 253-266.

[4] M. Giaquinta And M.-C. Hong, Partial regularity of minimizers of a functional ivolving forms and maps, Nonlinear differ. equ. appl., 11 (2004), pp. 469-490.

[5] M. Giaquinta and G. Modica, Regularity results for some classes of higher order non-linear elliptic systems, J. Reine Angew. Math., 311/312 (1979), pp. 145-169.

[6] M. Giaquinta And M. Struwe, On the partial regularity weak solutions of non-linear parabolic systems, Math. Z, 179 (1982), pp. 437-451.

[7] D. Gilbarg and N. S. Trudinger, Elliptic differential equations of second order, SpringerVerlag, 1983.

[8] E. Giusti And M, Miranda, Sulla regolarita delle soluzioni deboli di una class di sistemi ellittici quasilinerari, Arch. Rat. Mech. Anal., 31 (1968), pp. 173-184.

[9] J. Jost, Nonliear methods in Riemannian and Kählerian Geometry, Birkhäuser, Basel, 1988.

[10] O. A. Ladyzhenskaya, V. A. Solonnikov and N. N. Ural'Ceva, Linear and qusilinear equations of parabolic type, Tanslations of Mathematical Monographs 23. Providence, Rhode Island: American Mathematical Society, 1968.

[11] L. D. Landau And E.M. Lifshitz, Electrodynamics of Continuous Media, Pergmon Press, 1960.

[12] M. Struwe, On the Hölder continuity of bounded weak solutions of quasilinear parabolic systems, Manuscripta Math., 35 (1981), pp. 125-145.

[13] H-M YiN, Global solutions of Maxwell's equations in an electromagnetic field with a temperature dependent electrical conductivity, European journal of Applied Mathemtics, 5 (1994), pp. $57-64$.

[14] H-M Y IN, Regularity of solutions to Maxwell's system in quasi-stationary electromagnetic fields and application, Commun. in Partial Differential Equations, 22 (1997), pp. 1029-1053. 
[15] F. W. Warner, Foundations of Differentiable Manifolds and Lie Groups, Birkhäuser, Basel, 1988. 
M.-C. HONG, Y. TONEGAWA AND A. YASSIN 